\title{
Comparison of 2 Perioperative Management Protocols and Their Influence on Postoperative Recovery after Cytoreductive Surgery and Hyperthermic Intraperitoneal Chemotherapy: Standard Parenteral Nutrition, Selective Bowel Decontamination and Suprapubic Catheters?
}

\author{
Fortuné M.K. Elekonawo ${ }^{a} \quad$ Manon M.D. van der Meeren ${ }^{a}$ Geert A. Simkens ${ }^{b}$ \\ Johannes H.W. de Wilt ${ }^{a}$ Ignace H. de Hingh ${ }^{b}$ Andreas J.A. Bremers ${ }^{a}$ \\ ${ }^{a}$ Department of Surgery, Radboud University Medical Centre, Nijmegen, The Netherlands; \\ ${ }^{b}$ Department of Surgical Oncology, Catharina Hospital, Eindhoven, The Netherlands
}

\section{Keywords}

Postoperative recovery · Hyperthermic intraperitoneal .

Chemotherapy · Perioperative care protocol

\begin{abstract}
Background: Cytoreductive surgery and hyperthermic intraperitoneal chemotherapy (CRS-HIPEC) is associated with considerable postoperative morbidity, including ileus and infectious complications. Perioperative care is believed to be an important factor for the development and treatment of postoperative morbidity. Patients and Methods: Data on case-matched patients from a retrospective database of 2 Dutch HIPEC centres was compared. Patient selection and procedures were identical in both hospitals although perioperative management items differ slightly. In centre B, immediate total parenteral nutrition (TPN), suprapubic urine bladder catheter placement (SPCs) and selective decontamination of the digestive-tract are standard care for CRS-HIPEC patients, while in centre $A$, they are not. Results: From a total of 223 patients, 68 consecutive patients from centre B were
\end{abstract}

compared to 68 matched patients from centre A. TPN was administered to $54.4 \%$ of patients in centre $A$ because of prolonged ileus, whereas it was standard of care in centre B. In all, 105 (77.2\%) patients experienced postoperative complications including $17.6 \%$ who had a grades III-IV complication. The incidence of grade III-V complications was 18 (26.4\%) in centre A and $8(11.8 \%)$ in centre B $(p=0.03)$. Median hospital stay was 12 days (7-84) in $A$ and $11(6-80)$ in centre B $(p=0.546)$. Conclusions: Gastrointestinal recovery after CRS-HIPEC seems to take longer as compared to other surgical procedures. Between the 2 centres, a significant difference in severe complications was found, while standard TPN, selective bowel decontamination and SPCs were the only identified differences in perioperative care.

(c) 2018 The Author(s)

Published by S. Karger AG, Basel

F.M.K.E. and M.M.D.M. equally contributed to this work.

The results of this study were presented at the 10th International Congress on Peritoneal Surface Malignancies, Washington DC, USA, held from 17 to 19 November 2016.

\begin{tabular}{ll}
\hline KARGER & $\begin{array}{l}\text { ( } 2018 \text { The Author(s) } \\
\text { Published by S. Karger AG, Basel }\end{array}$ \\
E-Mail karger@karger.com & $\begin{array}{l}\text { This article is licensed under the Creative Commons Attribution- } \\
\text { NonCommercial-NoDerivatives 4.0 International License (CC BY- } \\
\text { NC-ND) (http://www.karger.com/Services/OpenAccessLicense). } \\
\text { Usage and distribution for commercial purposes as well as any dis- } \\
\text { tribution of modified material requires written permission. }\end{array}$
\end{tabular}

Fortuné M.K. Elekonawo

Department of Surgery

Radboud University Nijmegen Medical Centre

PO Box 9101, NL-6500 HB Nijmegen (The Netherlands)

E-Mail fortune.elekonawo@ radboudumc.nl 


\section{Introduction}

Globally, colorectal cancer is the third most common cancer in males and the second in females [1]. The advanced stage of disease is present in approximately $20 \%$ of patients at first presentation [2], including 4-7\% presenting with synchronous peritoneal carcinomatosis (PC) [3]. Patients with advanced $\mathrm{T}$ and $\mathrm{N}$ stage, poor tumour differentiation grade, mucinous tumours, younger age and right sided localization of the primary tumour have a higher risk for PC [3-5]. PC of colorectal origin has always been associated with poor prognosis and early studies in which patients were treated with palliative chemotherapy, reported median survival ranging from 3.1 to 7.0 months [5, 6]. Later studies indicate that modern chemotherapy regimens can increase survival up to a median of 12.5 months [7]. Complete cytoreductive surgery (CRS) and hyperthermic intraperitoneal chemotherapy (HIPEC) have demonstrated to significantly increase survival in selected patients with PC of colorectal origin [8-11]. Postoperative recovery in these patients is slower and associated with more morbidity compared to other abdominal procedures [12].

In elective colorectal surgery, fast track recovery programs that adhere to the 'enhanced recovery after surgery' (ERAS) principles [13, 14], have been demonstrated to be safe and effective $[15,16]$. ERAS programs in colorectal surgery cause faster recovery and fewer complications $[17,18]$. While patients operated for conventional colorectal, liver or gastro-intestinal tumours do benefit from its effectiveness, the distinctive postoperative course after CRS and HIPEC does not allow the application of standard ERAS protocols. This is not surprising, since the procedure is usually much longer and leads to extensive peritoneal trauma. Prolonged postoperative ileus (POI) frequently occurs after HIPEC. POI hinders patient recovery and increases postoperative morbidity, hospital costs, readmission rates and postoperative hospital stay (HS) [19-22]. Patients with PC of colorectal cancer treated with CRS and HIPEC in 2 centres between 2010 and 2015 were case-matched and analysed retrospectively. Both hospitals share identical patient selection standards and operative procedures in accordance with the nationwide protocol. The perioperative management however is different on 3 major items. These are the standard use of total parenteral nutrition (TPN), selective decontamination of the digestive tract (SDD) and suprapubic bladder catheters (SPCs) in one of the centres.

Comparison of 2 Different Perioperative

Management Protocols
The aim of the current study was to gain insight into early gastrointestinal recovery and perioperative morbidity in this specific group of patients and to evaluate the effect of 2 different perioperative care protocols on these outcomes.

\section{Patients and Methods}

\section{Patients}

Complete details on consecutive patients with PC of colorectal origin treated with CRS and HIPEC in Radboudumc Nijmegen and Catharina Ziekenhuis Eindhoven between 2010 and 2015 were available for this study. Exclusion criteria were nonprimary HIPEC procedures or other origins, for example, mesothelial origin. A total of 68 consecutive patients from Radboudumc Nijmegen were included and compared with 68 best matching patients selected from the 155 consecutive patients available from Catharina Ziekenhuis Eindhoven. Relevant patient-, tumour-, recovery- and treatment (procedure)-related data were collected in a retrospective database. Matching variables were age at time of surgery, BMI, metachronous or synchronous peritoneal carcinomatosis and peritoneal cancer index (PCI). After matching, a total of 136 patients were analysed. Data acquisition and analysis were approved by the local Ethics Committee.

\section{HIPEC Centres}

Both institutes involved in this study are Dutch HIPEC referral centres located in the southern region of The Netherlands. Indications for treatment with CRS and HIPEC and the applied operative techniques are identical and based on national consensus. Perioperative care protocols for these patients are identical in both hospitals except for 3 items: SDD, perioperative placement of suprapubic catheters and TPN started on the first day after operation are standard in centre B. In the other centre, Foley type urethral catheters are the standard of care and TPN and SDD are not included in the standard care protocol. These differences provided the opportunity to evaluate gastrointestinal recovery and complications as related to standard TPN plus SDD and suprapubic urine bladder catheters versus on-demand TPN, no SDD and transurethral catheters.

\section{Standard TPN Protocol}

The nutritional needs of all patients in centre B were calculated on an individual patient level to provide adequate TPN. A dietician used the revised Harris and Benedict equation to calculate base caloric demand [23]. To reach adequate caloric intake for surgically treated patients, a surplus of $30-50 \%$ was added to the outcome of the equation.

\section{Antibiotic prophylaxis}

Centre A administered an i.v. dose of 2,000 mg cefazolin and $500 \mathrm{mg}$ metronidazole $30 \mathrm{~min}$ prior to incision; adequate dosing of profylaxis was continued for the duration of the procedure. In centre $\mathrm{B}$, the same dose of metronidazole was administered $30 \mathrm{~min}$ prior to incision. On the day prior to the procedure, patients received an intravenous dose of 2,000 mg 
Ceftriaxone at $10 \mathrm{pm}$ Infectious complications that required additional antibiotic treatment were treated according to local protocols.

\section{SDD in Centre B}

For SDD, additional to the prophylaxis mentioned above, 1,000 mg Tobramycin/Colistin/Amphotericin-B oromucosal paste was applied 4 times per day, complemented by a $10-\mathrm{mL}$ gastrointestinal solution until oral food intake was resumed. SDD was used according to local protocol and is considered a valuable addition of antibiotic treatment to reduce the risk of infections after colorectal surgery $[24,25]$.

\section{Urine Catheters}

In centre B, SPCs are routinely placed during the first stage of the CRS-HIPEC procedure.

\section{HIPEC and CRS}

All procedures were performed by a specialized surgical team with ample experience in HIPEC. Leading HIPEC surgeons from both teams have crossed over to the other centre to participate in CRS-HIPEC surgery. All surgeons were directly or indirectly trained by the Netherlands Cancer Institute surgical team and therefore adhere to identical surgical techniques and procedures. Surgeons from both centres operated cases together in both hospitals. Peritoneal tumour extensiveness was scored using the PCI $[26,27]$.

Peritonectomy procedures were performed according to principles previously described by Sugarbaker [26]. Standard total omentectomy was part of all cytoreduction procedures that were performed in both centres. The gastro-omental arcade, previously known gastro-epiploic arcade, is often part of the omental resection specimen. In several cases, the arcade was spared.

The aim of each procedure was to achieve complete macroscopic resection, which was scored using the CC-score (CC0: complete macroscopic resection, CC1: residual tumour nodules $<2.5 \mathrm{~mm}, \mathrm{CC} 2$ : residual tumour nodules larger than $2.5 \mathrm{~mm}$ ). All procedures were performed as open "coliseum" surgery. For HIPEC with Mitomycine C, a solution of $35 \mathrm{mg} / \mathrm{m}^{2}$ (maximum $70 \mathrm{mg}$ ) in $0.9 \% \mathrm{NaCl}$ was used to perfuse the abdomen at a temperature of $42-43^{\circ} \mathrm{C}$ for $90 \mathrm{~min}$. In patients perfused with oxaliplatin, systemic chemotherapy with leucovorin and 5-fluorouracil (20 and $400 \mathrm{mg} / \mathrm{m}^{2}$ ) was administered prior to HIPEC. Oxaliplatin $460 \mathrm{mg} / \mathrm{m}^{2}$, in $5 \%$ dextrose $42-43^{\circ} \mathrm{C}$ was used for 30 min perfusion of the peritoneal cavity at $42-43^{\circ} \mathrm{C}$. During the historic period from 2010 to 2015 of the study, the treatment protocol was revised and changed from Mitomycine $\mathrm{C}$ to oxaliplatin in 2014 , in accordance with the national protocol update.

\section{Postoperative Recovery}

The date of the HIPEC procedure was defined as day 0. Multiple characteristics were registered as indicators of postoperative gastro-intestinal status: relief of nasogastric tube (used for gastric drainage), first day of tolerance of oral nutrition (not being clear fluids), first stool, start- and termination of TPN. Relief of nasogastric tube was defined as the day on which the tube was removed, provided no replacement in the following days during hospitalization was necessary. Oral tolerance was considered to be present when a patient experienced no nausea or vomiting after oral intake other than water or transparent liquid. First stool was defined as the day of the first defecation or enterostomy production (other than the commonly observed serous or postoperative early production on day $0-1$ ). Termination of TPN was defined by the last day a patient received any amount of intravenous nutrition.

\section{Complications}

Complications were scored using the Clavien Dindo complication classification [28]. Since TPN was part of the care protocol in centre B, TPN was excluded as complication criterium to score complications.

\section{Statistical Analyses}

Statistical analyses were performed using the Statistical Package for Social Sciences, version 22.0 (IBM Corp., Armonk, NY, USA). Comparisons of means and medians, respectively, were conducted with Student $t$ test or Mann-Whitney U test depending on distribution. Categorical variables were cross-tabulated and significance was determined by a chi-square test or Fisher's exact test according to sample size.

Pearson correlation was used to identify correlated variables. Correlations were tested 1 -sided with a $p$ value of 0.05 . All other tests were performed 2 sided and a significance level of $<0.05$ was used to reject the null hypothesis. For all calculations, cases in which essential data missed were excluded from analysis.

\section{Results}

A total of 223 patients treated in both hospitals between 2010 and 2015 with primary CRS and HIPEC for colorectal PC were included. All 68 elegible patients from centre $\mathrm{B}$ were included and compared with the 68 best matching patients from centre A. Table 1 summarizes general characteristics. After matching, no significant differences could be demonstrated in patient and tumour characteristics of patients in both groups.

Complete cytoreduction (CC0) was achieved in 95.6\% of treated patients. Procedure time was shorter in centre $A$ than that in B with 367 and 417 min respectively $(p<0.001)$. Patients treated in B had a higher estimated intraoperative loss of blood volume $(p<0.001)$; this however did not result in a difference in postoperative blood haemoglobin levels. Mitomycin C and oxaliplatin protocols were used in 115 patients $(84.6 \%)$ and 21 patients (15.4\%) respectively. All patients who were treated with oxaliplatin had surgery in centre B. Total omentectomy including the gastro-omental arcade was performed in 25 patients $(18.4 \%)$ in centre B. In 23 (16.9\%) centre $\mathrm{B}$ patients, the gastro-omental arcade was spared and in the remaining 88 patients of $(64.7 \%)$ the arcade status was not unequivocally described. Time to removal of nasogastric tube and oral tolerance of food was not statistically different in these groups $(p=0.933$ and $p=0.633$ ). 
Table 1. Characteristics of patients, primary tumours and treatment procedures

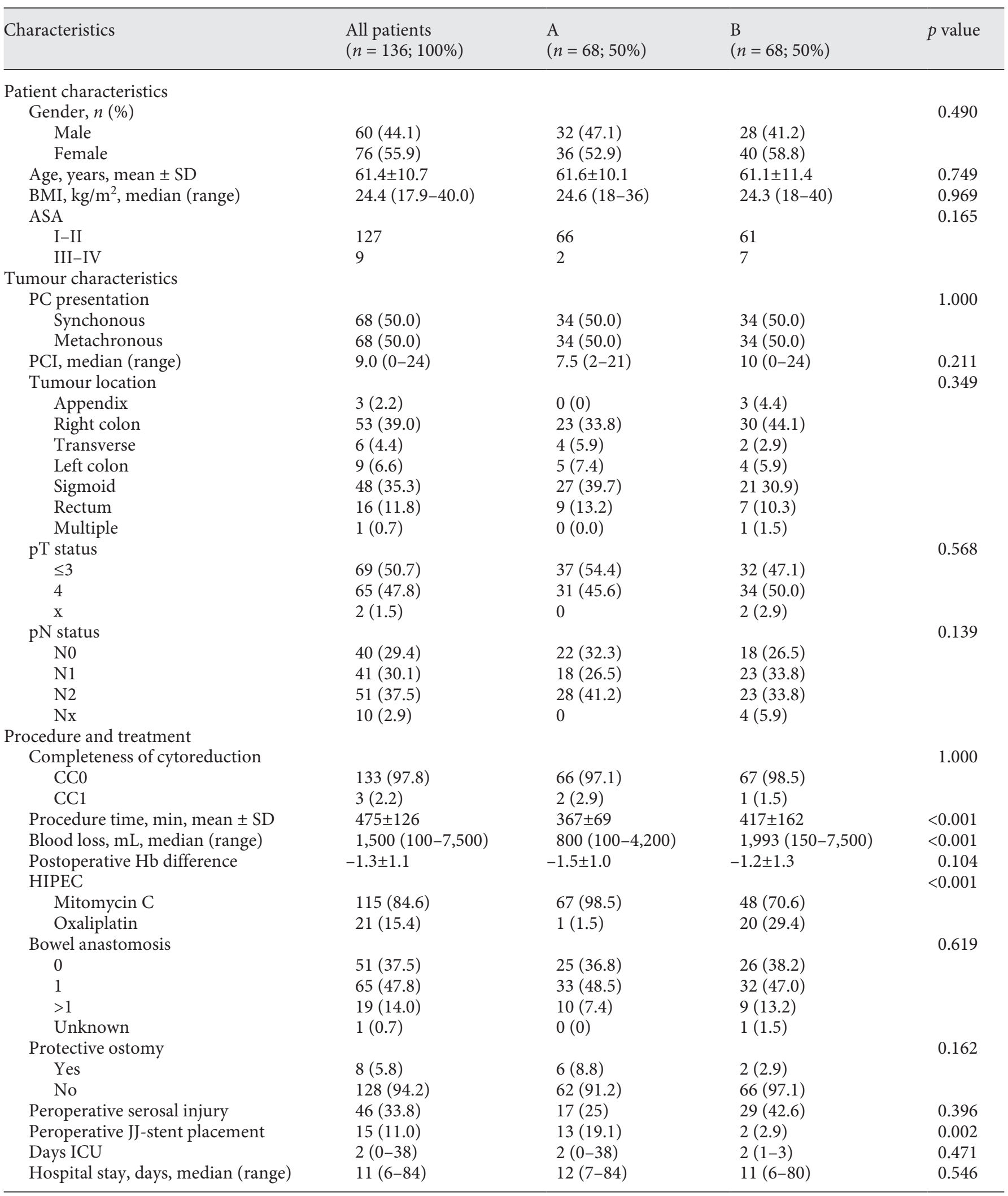

PC, peritoneal cancer; PCI, peritoneal cancer index; ICU, intensive care unit. 
Table 2. Complications per hospital

\begin{tabular}{|c|c|c|c|c|}
\hline Complications & $\begin{array}{l}\text { All patients } \\
(n=136 ; 100 \%)\end{array}$ & $\begin{array}{l}\text { A } \\
(n=68 ; 50 \%)\end{array}$ & $\begin{array}{l}\mathrm{B} \\
(n=68 ; 50 \%)\end{array}$ & $p$ value \\
\hline Any complication & $105(77.2)$ & $48(70.6)$ & $57(83.8)$ & 0.066 \\
\hline Uncomplicated & $31(22.8)$ & $20(29.4)$ & $11(16.2)$ & \\
\hline Clavien Dindo score & & & & 0.028 \\
\hline I & $17(12.5)$ & $8(11.8)$ & $9(13.2)$ & \\
\hline II & $62(45.6)$ & $22(32.4)$ & $40(58.8)$ & \\
\hline III & $15(11.0)$ & $10(14.7)$ & $5(7.4)$ & \\
\hline IV & $9(6.6)$ & $6(8.8)$ & $3(4.4)$ & \\
\hline $\mathrm{V}$ & $2(1.5)$ & $2(2.9)$ & $0(0.0)$ & \\
\hline Any infection & $42(30.9)$ & $29(42.6)$ & $13(19.1)$ & 0.003 \\
\hline Wound & $24(17.6)$ & $17(25.0)$ & $7(10.3)$ & 0.024 \\
\hline Urinary tract & $11(8.1)$ & $9(13.2)$ & $2(2.9)$ & 0.028 \\
\hline Line & $5(3.7)$ & $\mathrm{N}$ & $5(7.4)$ & - \\
\hline Other & $13(9.6)$ & $10(14.7)$ & $3(4.4)$ & 0.041 \\
\hline Intra-abdominal & $20(14.7)$ & $14(20.6)$ & $6(8.8)$ & 0.053 \\
\hline Other & $8(5.9)$ & $1(1.5)$ & $7(10.3)$ & 0.062 \\
\hline
\end{tabular}

$\mathrm{N}$, not scored or described.

\section{Complications}

A complicated postoperative course was observed in $105(77.2 \%)$ of all patients and $24(17.6 \%)$ had grades III-IV Clavien-Dindo complications (Table 2).

Grades III-V were more commonly observed in A: $n=$ $18(26.4 \%)$ vs. $n=8(11.8 \%)$ in B $(p=0.03)$. Most common grades III-IV complications were anastomotic leakages, fistulas and intra-abdominal abscesses.

Infectious complications were more prominent in centre A - $29(42.6 \%)$ vs. $13(19.1 \%)$ in B ( $p=0.003)$ - including a difference in the incidence of wound-, urinary tract-, other infections and the incidence of anastomotic leakage. In centre $\mathrm{B}$, fewer urinary tract infections occurred: $n=2(2.9 \%)$ vs. $n=9(13.2 \%)$ in centre A $(p=$ 0.028). In centre $A, 13$ (19.1\%) patients had a double J stent placed preoperatively. Of those patients, 5 (38.5\%) developed an infection of the urinary tract. The numbers for the whole study were 15 and 5, respectively (33\%). Median hospital stay was not statistically different between the centres: 12 days (7-84) in A and $11(6-80)$ in B $(p=0.546)$.

A higher PCI was related with a longer HS $(p=0.03)$. And a longer HS was moderately correlated with oral intolerance $(r=0.632, p<0.001)$.

\section{Standard TPN and TPN on Demand}

TPN was administered per protocol in $98.5 \%$ (1 patient did not receive TPN due to line failure) of patients in centre B. In centre A, $54.4 \%$ of the patients had an indication to administer TPN and were treated accordingly (Table 3). The duration of TPN was longer in A with a median of 10 $(4-65)$ vs. $8(0-28)$ days postoperatively in B $(p=0.03)$. Generally, TPN was ended before discharge. The day of first oral tolerance and nasogastric tube removal was 1 day earlier in $\mathrm{A}(p=0.03, p<0.001)$, but in $\mathrm{A}$, nasogastric tubes were reinserted more frequently: $n=18$ vs. $n=7$ ( $p=$ 0.018 ). First stool occurred on day 5 in both groups and abdominal wound drains were removed earlier in $\mathrm{A}$ with median day 3 vs. 7 in $\mathrm{B}(p<0.001)$.

\section{Discussion}

CRS combined with HIPEC is a curative multimodality approach for peritoneal carcinomatosis with a relatively high rate of morbidity and delayed return of gastrointestinal functions compared to other abdominal surgical procedures. Postoperative complications and gastrointestinal recovery were carefully registered in 2 Dutch tertiary referral hospitals for this surgical technique. Despite central intravenous catheter-related postoperative morbidity, a postoperative protocol with standard SDD, TPN and SPCs showed a decreased incidence of severe postoperative morbidity. The incidence of delayed return of gastrointestinal function was remarkably high compared to what is described for other gastrointestinal surgical procedures [29-32]. 
Table 3. Parameters of gastrointestinal status

\begin{tabular}{lccrr}
\hline Gastrointestinal status & $\begin{array}{l}\text { All patients } \\
(n=136 ; 100 \%)\end{array}$ & $\begin{array}{l}\text { A } \\
(n=68 ; 50 \%)\end{array}$ & $\begin{array}{l}\text { B } \\
(n=68 ; 50 \%)\end{array}$ & $p$ value \\
\hline TPN, $n(\%)$ & $104(77)$ & $37(54.4)$ & $67(98.5)$ & $<0.001$ \\
TPN start, days & $1(0-23)$ & $3.5(0-23)$ & $1(0-3)$ & $<0.001$ \\
TPN stop, days, median (range) & $8.0(0-65)$ & $10(4-65)$ & $8(2-28)$ & 0.043 \\
TPN total, days & $7(1-61)$ & $5(1-61)$ & $7(2-20)$ & 0.243 \\
NT stop, days, median (range) & $3(0-71)$ & $3(0-10)$ & $4(1-71)$ & 0.002 \\
NT reinsertion, $n$ (\%) & $25(18.4)$ & $18(26.5)$ & $7(10.3)$ & 0.018 \\
First stool, days, median (range) & $5(1-12)$ & $5(1-11)$ & $5(1-12)$ & 0.430 \\
OT, days, median (range) & $5(1-76)$ & $5(1-17)$ & $6(2-76)$ & 0.034 \\
AWD stop, days, median (range) & $5(2-20)$ & $3(2-10)$ & $7(2-20)$ & $<0.001$ \\
First stool $>$ day 4, $n(\%)$ & $68(50)^{*}$ & $34(50)$ & $34(50)$ & 0.924 \\
First OT $>$ day 4, $n$ (\%) & $76(55.9)^{* *}$ & $32(47.1)$ & $44(64.7)$ & 0.019 \\
\hline
\end{tabular}

* 9 not described; ** 13 not described.

TPN, total parenteral nutrition; NT, nasogastric tube; OT, oral tolerance; AWD, abdominal wound drains.

To minimize selection bias and create a representative patient group, all consecutive patients over 5 years from one centre were included and matched for all known important risk factors with patients from the larger cohort of the other centre.

The difference in medication used for the perfusion between the centres differed between study groups. However, available studies comparing the results of mitomycin $\mathrm{C}$ with oxaliplatin in the setting of HIPEC have shown very similar results with respect to postoperative complications [3335].

\section{Complications and Infections}

The total complication rate was higher in centre B, but severe complications were more common in centre A. Differences in perioperative care protocols might have an effect on the type of complications and complication rates, but the retrospective nature of this study prevents any direct association. There was a substantial difference between the centres considering the time abdominal drains were kept, but a recent meta-analysis showed that early drain removal after abdominal surgery is neither beneficial nor detrimental when related to infection rates [36]. In pelvic surgery, a lower incidence of urinary tract infections was previously described with the use of SPCs over transurethral catheters [37]. More urinary tract infections were found in centre A, where urethral catheters were used as standard of care. However, the incidence of double-J stents was also higher in this population. J-J stents were placed when indicated after distal ureteral reimplantations or reconstructions. In the total cohort, $45 \%$ of patients with a urinary tract infection $(n=11)$ had a double-J stent $(n=$ 5 ), suggesting a higher susceptibility for urinary tract infections in these patients. The effect of urethral- or suprapubic catheters in combination with double-J-stents remains unclear because of the limited number of patients with double J stents.

\section{Gastrointestinal Recovery}

Gastrointestinal recovery seems to take more time in CRS and HIPEC procedures than in other fields of abdominal surgery [29-32]. Although we observed a relation between PCI and hospital stay in the total cohort, differences in postoperative recovery did not seem to correspond with differences in length of postoperative stay between the centres. After major abdominal surgical procedures, patients may experience severe nausea and vomiting after oral ingestion of food for several days postoperatively. In those cases, patients are at risk of aspiration, dehydration or malnutrition. Relief of symptoms may be achieved by nasogastric tube placement and additional TPN for nutritional purposes. Reinsertion of nasogastric tubes was more often necessary in group A, which could be due to premature removal postoperatively.

Enteral nutrition is suggested to be the preferred option whenever adequately possible, since it is associated with fewer (septic) complications, reduced costs, lower incidence of ileus or anastomotic leakage and a shorter hospital stay [38-41]. Also, a better perioperative nutritional status is associated with less number of complications in gastro-intestinal surgery patients [42] and the patient with peritoneal carcinomatosis is often in sub- 
optimal nutritional status even before surgery. TPN is standard of care in centre B, as opposed to the other centre where it is used when oral intake of food and calories was insufficient after several days. The number of days to oral tolerance and nasogastric tube relief was smaller in centre A, which might be explained by the early enteral feeding policy and a more aggressive policy in removing nasogastric tubes and enteral feeding. The difference in time to oral tolerance, however, did not result in a difference in hospital stay. Theoretically a better nutritional status resulting from TPN could reduce the susceptibility for infections, although this remains unclear. The present study shows that TPN was still a necessity in over half of patients to ensure adequate nutrition intake, while an early enteral feeding policy is supposed to result in an earlier oral tolerance for food. Ideally clinicians should be able to determine which specific patients would benefit from different feeding policies. However, the exploratory setup of this study does not allow a fair comparison of TPN versus early enteral feeding.

\section{Conclusions}

The current exploratory study provides insight into the duration of recovery of the gastrointestinal tract in CRS and HIPEC patients. Significantly less severe infectious complications were observed in a group of CRS and HIPEC patients treated with a care protocol that involved standard TPN, SDD and SPCs. Moreover, TPN was unavoidable in a large part of CRS and HIPEC patients with an early enteral feeding policy.

The study shows a significant difference in relevant early outcome of the treatment. The differences between care protocols might well explain these differences on theoretical grounds. The burden of these complications for patients and hospital finance demands prospective evaluation in a multicentre study.

\section{Disclosure Statement}

None of the authors have any conflicts of interest to disclose.

\section{References}

1 Torre LA, Bray F, Siegel RL, Ferlay J, LortetTieulent J, Jemal A: Global cancer statistics, 2012. Cancer J Clin 2015;65:87-108.

2 van der Geest LG, Lam-Boer J, Koopman M, Verhoef C, Elferink MA, de Wilt JH: Nationwide trends in incidence, treatment and survival of colorectal cancer patients with synchronous metastases. Clin Exp Metastasis 2015;32:457-465.

3 Lemmens VE, Klaver YL, Verwaal VJ, Rutten HJ, Coebergh JW, de Hingh IH: Predictors and survival of synchronous peritoneal carcinomatosis of colorectal origin: a populationbased study. Int J Cancer 2011;128:27172725.

4 Segelman J, Granath F, Holm T, Machado M, Mahteme $\mathrm{H}$, Martling A: Incidence, prevalence and risk factors for peritoneal carcinomatosis from colorectal cancer. Br J Surg 2012;99:699-705.

5 Jayne DG, Fook S, Loi C, Seow-Choen F: Peritoneal carcinomatosis from colorectal cancer. Br J Surg 2002;89:1545-1550.

6 Sadeghi B, Arvieux C, Glehen O, Beaujard AC, Rivoire M, Baulieux J, Fontaumard E, Brachet A, Caillot JL, Faure JL, Porcheron J, Peix JL, Francois Y, Vignal J, Gilly FN: Peritoneal carcinomatosis from non-gynecologic malignancies: results of the EVOCAPE 1 multicentric prospective study. Cancer 2000; 88:358-363.

7 Klaver YL, Leenders BJ, Creemers GJ, Rutten HJ, Verwaal VJ, Lemmens VE, de Hingh IH: Addition of biological therapies to palliative chemotherapy prolongs survival in patients with peritoneal carcinomatosis of colorectal origin. Am J Clin Oncol 2013;36:157-161.

8 Verwaal VJ, van Ruth S, de Bree E, van Sloothen GW, van Tinteren H, Boot H, Zoetmulder FA: Randomized trial of cytoreduction and hyperthermic intraperitoneal chemotherapy versus systemic chemotherapy and palliative surgery in patients with peritoneal carcinomatosis of colorectal cancer. J Clin Oncol 2003;21:3737-3743.

9 Glehen O, Cotte E, Schreiber V, Sayag-Beaujard AC, Vignal J, Gilly FN: Intraperitoneal chemohyperthermia and attempted cytoreductive surgery in patients with peritoneal carcinomatosis of colorectal origin. Br J Surg 2004;91:747-754.

10 Verwaal VJ, Bruin S, Boot H, van Slooten G, van Tinteren H: 8-year follow-up of randomized trial: cytoreduction and hyperthermic intraperitoneal chemotherapy versus systemic chemotherapy in patients with peritoneal carcinomatosis of colorectal cancer. Ann Surg Oncol 2008; 15:2426-2432.

11 Froysnes IS, Larsen SG, Spasojevic M, Dueland S, Flatmark K: Complete cytoreductive surgery and hyperthermic intraperitoneal chemotherapy for colorectal peritoneal metastasis in Norway: prognostic factors and oncologic outcome in a national patient cohort. J Surg Oncol 2016;114:222-227.

12 Baratti D, Kusamura S, Mingrone E, Balestra MR, Laterza B, Deraco M: Identification of a subgroup of patients at highest risk for com- plications after surgical cytoreduction and hyperthermic intraperitoneal chemotherapy. Ann Surg 2012;256:334-341.

13 Fearon KC, Ljungqvist O, Von Meyenfeldt M, Revhaug A, Dejong CH, Lassen K, Nygren J, Hausel J, Soop M, Andersen J, Kehlet H: Enhanced recovery after surgery: a consensus review of clinical care for patients undergoing colonic resection. Clin Nutr 2005;24:466477.

14 Lassen K, Soop M, Nygren J, Cox PB, Hendry $\mathrm{PO}$, Spies C, von Meyenfeldt MF, Fearon KC, Revhaug A, Norderval S, Ljungqvist O, Lobo $\mathrm{DN}$, Dejong CH: Consensus review of optimal perioperative care in colorectal surgery: enhanced recovery after surgery (ERAS) Group recommendations. Arch Surg 2009; 144:961-969.

15 Zhuang CL, Ye XZ, Zhang XD, Chen BC, Yu $Z$ : Enhanced recovery after surgery programs versus traditional care for colorectal surgery: a meta-analysis of randomized controlled trials. Dis Colon Rectum 2013;56:667-678.

16 Spanjersberg WR, Reurings J, Keus F, van Laarhoven CJ: Fast track surgery versus conventional recovery strategies for colorectal surgery. Cochrane Database Syst Rev 2011; 2:CD007635.

17 Gillissen F, Hoff C, Maessen JM, Winkens B, Teeuwen JH, von Meyenfeldt MF, Dejong $\mathrm{CH}$ : Structured synchronous implementation of an enhanced recovery program in elective colonic surgery in 33 hospitals in The Netherlands. World J Surg 2013;37:1082-1093. 
18 Teeuwen PH, Bleichrodt RP, Strik C, Groenewoud JJ, Brinkert W, van Laarhoven CJ, van Goor H, Bremers AJ: Enhanced recovery after surgery (ERAS) versus conventional postoperative care in colorectal surgery. J Gastrointest Surg 2010;14:88-95.

19 Li LT, Mills WL, White DL, Li A, Gutierrez AM, Berger DH, Naik AD: Causes and prevalence of unplanned readmissions after colorectal surgery: a systematic review and meta-analysis. J Am Geriatr Soc 2013; 61:1175-1181.

20 Mattei P, Rombeau JL: Review of the pathophysiology and management of postoperative ileus. World J Surg 2006;30:1382-1391.

21 Baig MK, Wexner SD: Postoperative ileus: a review. Dis Colon Rectum 2004;47:516-526.

22 Doorly MG, Senagore AJ: Pathogenesis and clinical and economic consequences of postoperative ileus. Surg Clin North Am 2012; 92:259-272, viii.

23 Roza AM, Shizgal HM: The Harris Benedict equation reevaluated: resting energy requirements and the body cell mass. Am J Clin Nutr 1984;40:168-182.

24 Abis GS, Stockmann HB, van Egmond M, Bonjer HJ, Vandenbroucke-Grauls CM, Oosterling SJ: Selective decontamination of the digestive tract in gastrointestinal surgery: useful in infection prevention? A systematic review. J Gastrointest Surg 2013;17:2172-2178.

25 Silvestri L, van Saene HK: Selective decontamination of the digestive tract: an update of the evidence. HSR Proc Intensive Care Cardiovasc Anesth 2012;4:21-29.

26 Sugarbaker PH: Peritonectomy procedures. Ann Surg 1995;221:29-42.

27 Jacquet P, Sugarbaker PH: Clinical research methodologies in diagnosis and staging of patients with peritoneal carcinomatosis. Cancer Treat Res 1996;82:359-374.
28 Dindo D, Demartines N, Clavien PA: Classification of surgical complications: a new proposal with evaluation in a cohort of $6336 \mathrm{pa}-$ tients and results of a survey. Ann Surg 2004; 240:205-213.

29 Pikarsky AJ, Saida Y, Yamaguchi T, Martinez S, Chen W, Weiss EG, Nogueras JJ, Wexner $\mathrm{SD}$ : Is obesity a high-risk factor for laparoscopic colorectal surgery? Surg Endosc 2002; 16:855-858.

30 Franko J, O'Connell BG, Mehall JR, Harper SG, Nejman JH, Zebley DM, Fassler SA: The influence of prior abdominal operations on conversion and complication rates in laparoscopic colorectal surgery. JSLS 2006;10:169175.

31 Petros JG, Realica R, Ahmad S, Rimm EB, Robillard RJ: Patient-controlled analgesia and prolonged ileus after uncomplicated colectomy. Am J Surg 1995;170:371-374.

32 Raue W, Haase O, Junghans T, Scharfenberg M, Muller JM, Schwenk W: "Fast-track" multimodal rehabilitation program improves outcome after laparoscopic sigmoidectomy: a controlled prospective evaluation. Surg Endosc 2004; 18:1463-1468.

33 van Eden WJ, Kok NFM, Woensdregt K, Huitema ADR, Boot H, Aalbers AGJ: Safety of intraperitoneal Mitomycin $C$ versus intraperitoneal oxaliplatin in patients with peritoneal carcinomatosis of colorectal cancer undergoing cytoreductive surgery and HIPEC. Eur J Surg Oncol 2018;44:220-227.

34 Tan GHC, Shannon NB, Chia CS, Soo KC, Teo MCC: Platinum agents and mitomycin C-specific complications in cytoreductive surgery (CRS) and hyperthermic intraperitoneal chemotherapy (HIPEC). Int J Hyperthermia 2017:1-6.

35 Hompes D, D'Hoore A, Wolthuis A, Fieuws S, Mirck B, Bruin S, Verwaal V: The use of
Oxaliplatin or Mitomycin C in HIPEC treatment for peritoneal carcinomatosis from colorectal cancer: a comparative study. J Surg Oncol 2014;109:527-532.

$36 \mathrm{Wu}$ X, Tian W, Kubilay NZ, Ren J, Li J: Is it necessary to place prophylactically an abdominal drain to prevent surgical site infection in abdominal operations? A systematic meta-review. Surg Infect 2016;17:730738.

37 Branagan GW, Moran BJ: Published evidence favors the use of suprapubic catheters in pelvic colorectal surgery. Dis Colon Rectum 2002;45:1104-1108.

38 Saito H, Trocki O, Alexander JW, Kopcha R, Heyd T, Joffe SN: The effect of route of nutrient administration on the nutritional state, catabolic hormone secretion, and gut mucosal integrity after burn injury. JPEN J Parenter Enteral Nutr 1987;11:1-7.

39 Ward N: Nutrition support to patients undergoing gastrointestinal surgery. Nutr J 2003;2: 18.

40 Bozzetti F, Braga M, Gianotti L, Gavazzi C, Mariani L: Postoperative enteral versus parenteral nutrition in malnourished patients with gastrointestinal cancer: a randomised multicentre trial. Lancet 2001;358:14871492.

41 Boelens PG, Heesakkers FF, Luyer MD, van Barneveld KW, de Hingh IH, Nieuwenhuijzen GA, Roos AN, Rutten HJ: Reduction of postoperative ileus by early enteral nutrition in patients undergoing major rectal surgery: prospective, randomized, controlled trial. Ann Surg 2014;259:649-655.

42 Kwag SJ, Kim JG, Kang WK, Lee JK, Oh ST: The nutritional risk is a independent factor for postoperative morbidity in surgery for colorectal cancer. Ann Surg Treat Res 2014; 86:206-211. 